Supporting Information

\title{
Electronic Structure and Transport Properties of
}

\section{Single-molecule Junctions with Different Sizes of $\pi$ -}

conjugated System

Yuji Isshiki, Tomoaki Nishino, and Shintaro Fujii

Department of Chemistry, Graduate School of Science and Engineering, Tokyo Institute of

Technology, 2-12-1 W4-10 Ookayama, Meguro-ku, Tokyo 152-8551, Japan

Table of contents

1. Theoretical simulations

2. Additional STM-BJ result

3. Analysis of the Junction Length

4. Statistical Analysis of $I-V$ Curves 


\section{Theoretical Simulations}

UV spectra were obtained with a solution containing approximately $0.1 \mathrm{mM}$ of the target molecules. They were measured using a JASCO V-650 with a wavelength range of 200-800 $\mathrm{nm}$ at $300 \mathrm{~K}$. The molecular orbitals in the optimized geometries and the theoretical UV-vis absorption spectra in the gap phase were calculated with the B3LYP/6-31G* level using the Gaussian 16 code. ${ }^{1-2}$ The orange lines shown in Figure S1 (A-C) correspond to the contributions of absorption strength. The energy and oscillator strength of the first and second major contributing orbitals are presented in Table S1. Figure S2 shows the molecular orbitals of the Py1, Qx2, and Ph3 isolated molecules.

The DFT calculations of Py1, Qx2, and Ph3 single-molecule junctions, sandwiched between two Au electrodes, were performed using cluster models where each side of the junction consisted of $20 \mathrm{Au}$ atoms in a similar manner to those in the previous study. ${ }^{3}$ Within the generalized gradient approximation (GGA) in the Perdew-Burke-Ernzerhof (PBE) form, ${ }^{4-5}$ the electronic wave functions were expanded in a double-numeric polarized basis set with a real-space cutoff of $0.4 \mathrm{~nm}$ using the Dmol3 code. ${ }^{6-7}$ We used geometry information from our previous DFT studies of Py1 single-molecule junctions. ${ }^{7}$ The dispersion correction method was used as an add-on to the standard Kohn-Sham density functional theory (DFT-D). ${ }^{8}$ We initially optimized the configurations of $\mathrm{Au} / \mathrm{Py} 1 / \mathrm{Au}$, $\mathrm{Au} / \mathrm{Qx} 2 / \mathrm{Au}$, and $\mathrm{Au} / \mathrm{Ph} 3 / \mathrm{Au}$ junctions with the electrode distance of $0.779 \mathrm{~nm}$ adopted from previous studies. ${ }^{3}$ Here, the two outer double Au layers of the left and right electrodes were fixed, and the other atomic positions were allowed degrees of freedom. The total energy profile as a function of the distance between two electrodes was obtained by decreasing the distance by $0.005 \mathrm{~nm}$ in a stepwise fashion; the geometry was relaxed in 
each step. Figure S3D show a profile of the total energy versus the electrode distance for Py1, in which two local energy minima were found, and the positions were denoted by $\mathrm{H}$ and L. Based on the local energy minima at the small and large electrode distances, the length of the molecular junctions in the $\mathrm{H}$ and $\mathrm{L}$ states are determined as shown in Figure S3E and F. The relaxed geometries for the L states of Qx2 and Ph3 were obtained on the basis of the relaxed geometry of Py1 in the L state. The Py1 molecule in the L state (Figure S3F) was replaced by a molecule of $\mathrm{Qx} 2$ and $\mathrm{Ph} 3$, and the junction geometries were relaxed. Then, the distance between two electrodes was decreased and increased by $0.005 \mathrm{~nm}$ in a stepwise fashion, and the geometry was relaxed in each step. The length of the molecule junctions of $\mathrm{Qx} 2$ and $\mathrm{Ph} 3$ in the L states was determined to be $0.78 \mathrm{~nm}$, at which local energy minima were achieved (Figure S4A). 

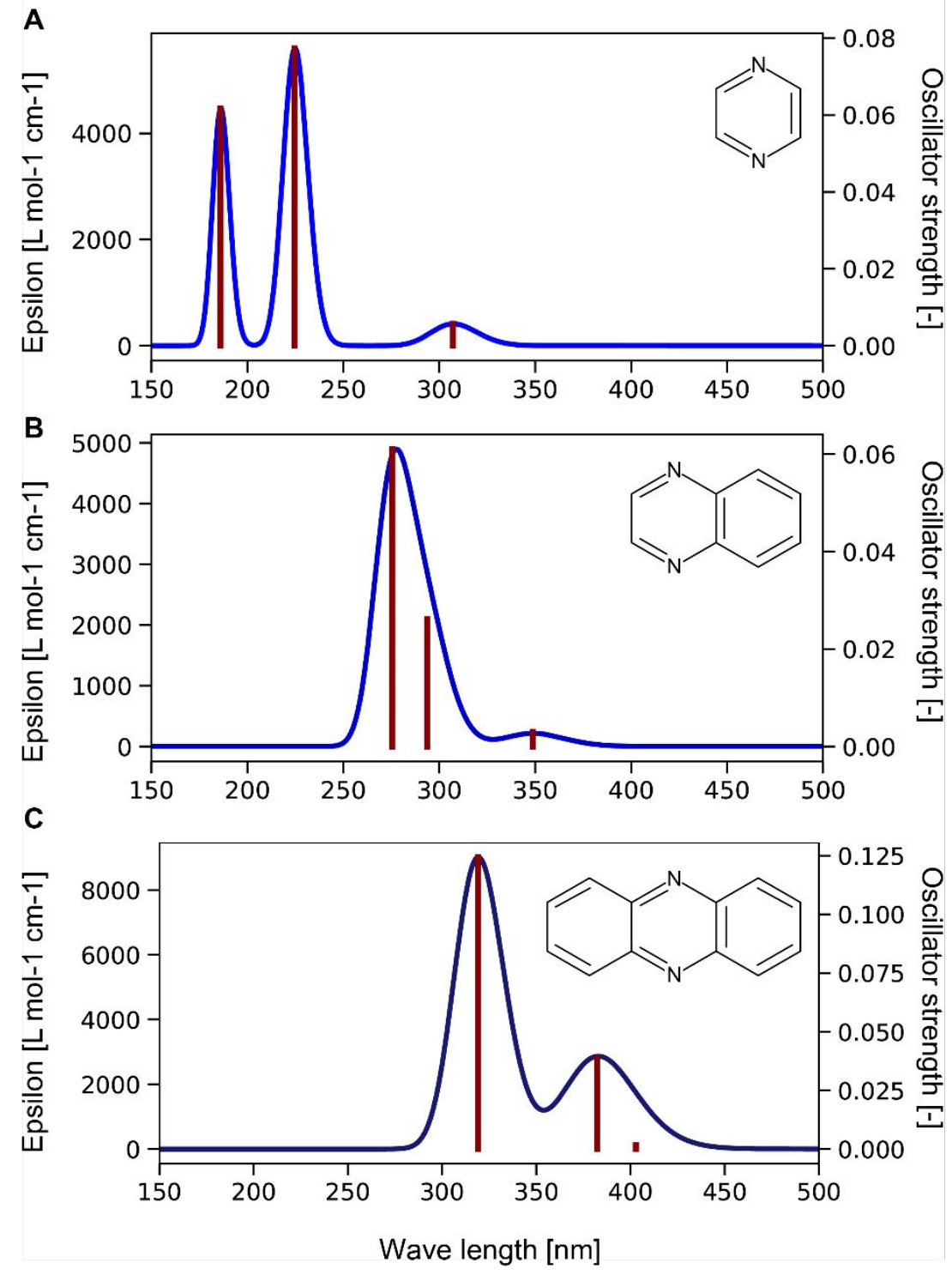

Figure S1. (A-C) Calculated absorption spectra using B3LYP/6-31G* for (A) Py1, (B) Qx2, and (C) $\mathrm{Ph} 3$ 
Table S1. Adsorption energy, wavelength, oscillator strength, and major contributions of the orbital to adsorption

\begin{tabular}{ccccc}
\hline Molecule & Energy [cm $\left.{ }^{-1}\right]$ & Wavelength [nm] & $\begin{array}{c}\text { Oscillator } \\
\text { strength }\end{array}$ & $\begin{array}{c}\text { Major } \\
\text { contributions }\end{array}$ \\
\hline Py1 / peak1 & 32558.2 & 307.1 & 0.0057 & $\pi \rightarrow \pi^{*}$ \\
Py1 / peak2 & 44505.7 & 224.6 & 0.0773 & $\mathrm{n} \rightarrow \pi^{*}$ \\
Qx2 / peak1 & 28671.4 & 348.8 & 0.003 & $\mathrm{n} \rightarrow \pi^{*}$ \\
Qx2 / peak2 & 34047.9 & 293.7 & 0.0261 & $\pi \rightarrow \pi^{*}$ \\
Ph3 / peak1 & 24823.3 & 402.8 & 0.0016 & $\mathrm{n} \rightarrow \pi^{*}$ \\
Ph3 / peak2 & 26154.9 & 382.3 & 0.0386 & $\pi \rightarrow \pi^{*}$ \\
\hline
\end{tabular}


A

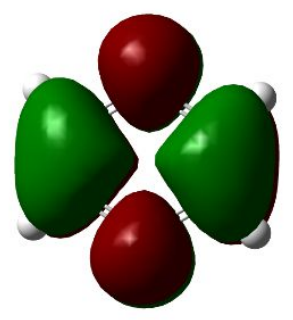

Py1 / LUMO

D

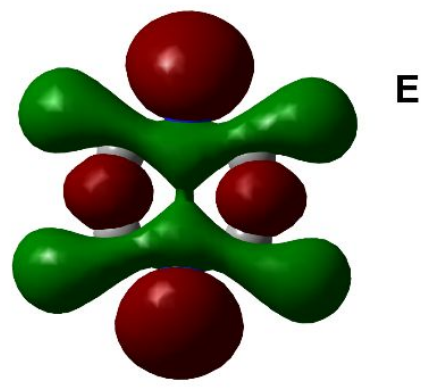

Py1 / HOMO

G

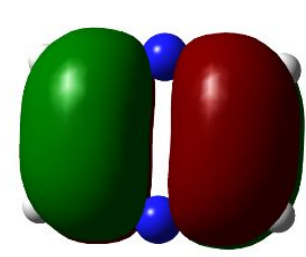

Py1 / HOMO-1
B

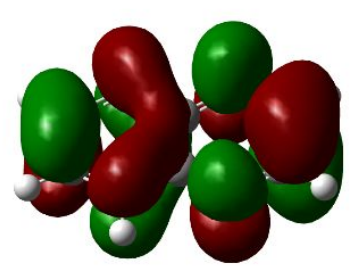

Qx2 / LUMO

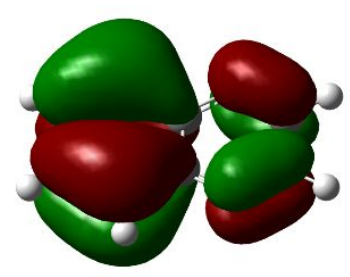

Qx2 / HOMO

H

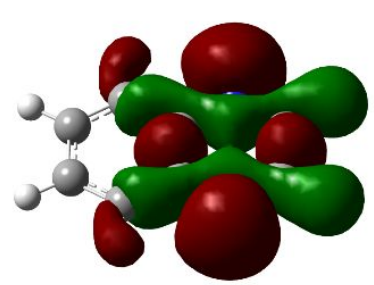

Qx2 / HOMO-1

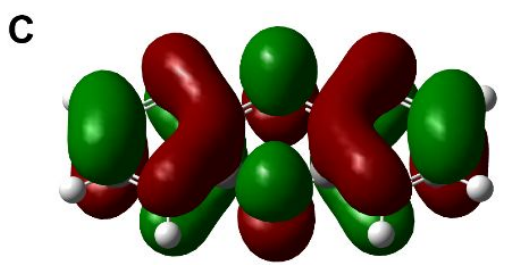

Ph3 / LUMO

F

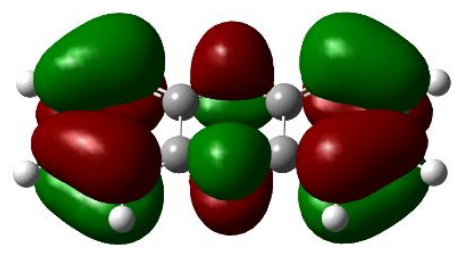

Ph3 / HOMO

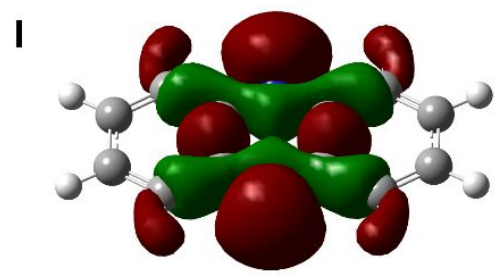

Ph3 / HOMO-1

Figure S2. Plots of (A-C) LUMO, (D-F) HOMO, and (G-I) HOMO-1 orbitals of Py1, Qx2, and Ph3 (Isovalue $=0.02$ e $/$ a.u. ${ }^{4}$ ) 

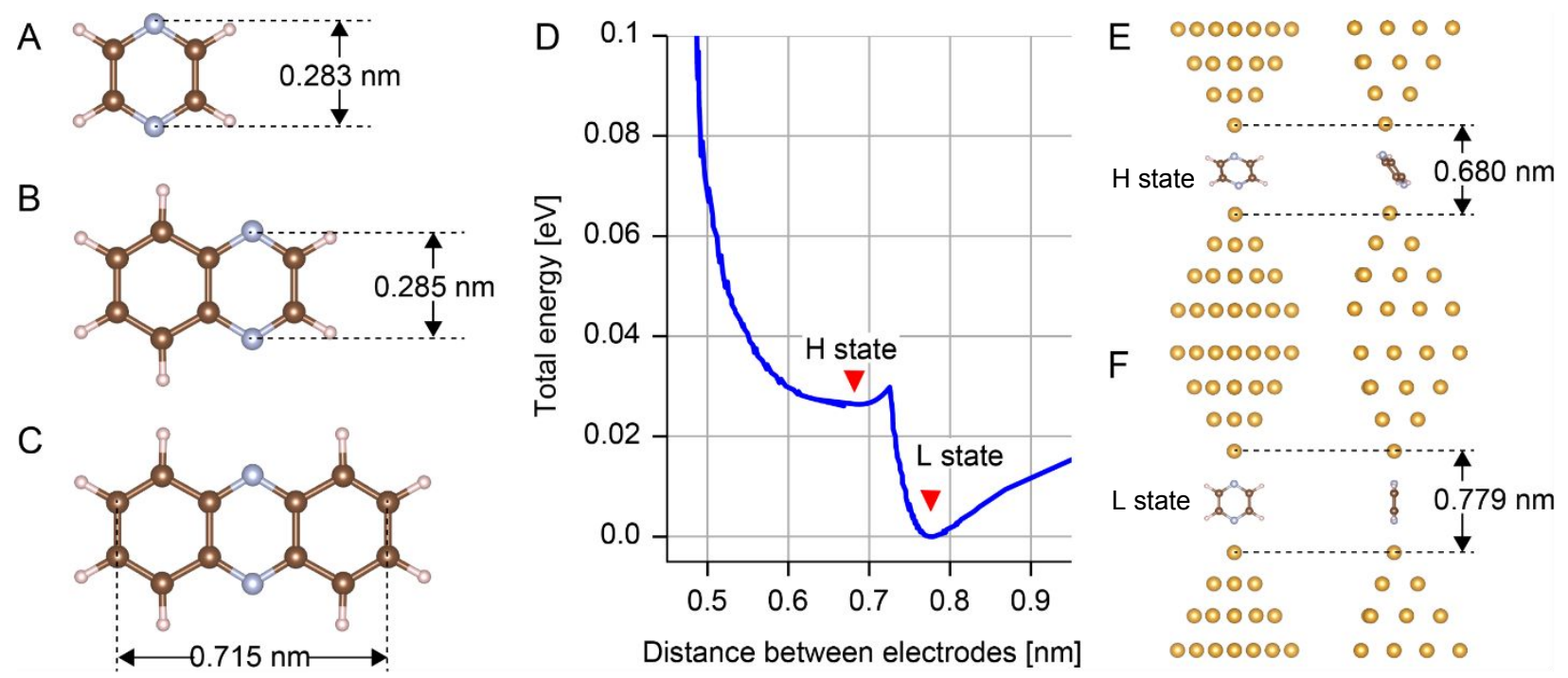

Figure S3. (A-C) Optimized geometries of Py1, Qx2, and Ph3 in a gas phase (D) Calculated total energy profile as a function of the distance between two electrodes for Py1 with the $\mathrm{Au}$ electrodes. The two local minima in the total energy profile are indicated by arrows, and denoted by the $\mathrm{H}$ and L states. (E, F) Optimized junction geometries for Py1 in the $\mathrm{H}$ and $\mathrm{L}$ states 
A

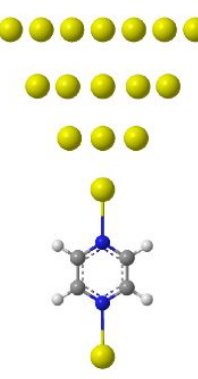

०००

00000

0000000

Au-Py1-Au

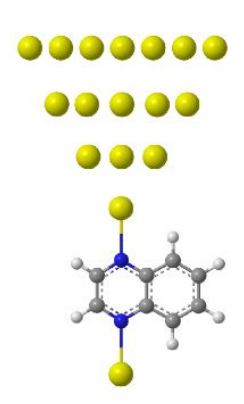

ค००

00000

0000000

Au-Qx2-Au

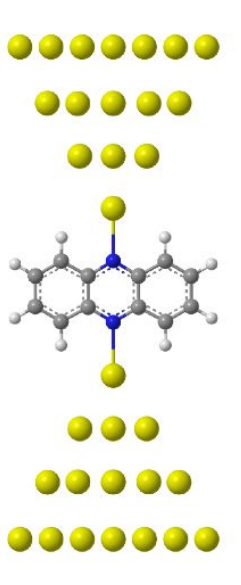

Au-Ph3-Au

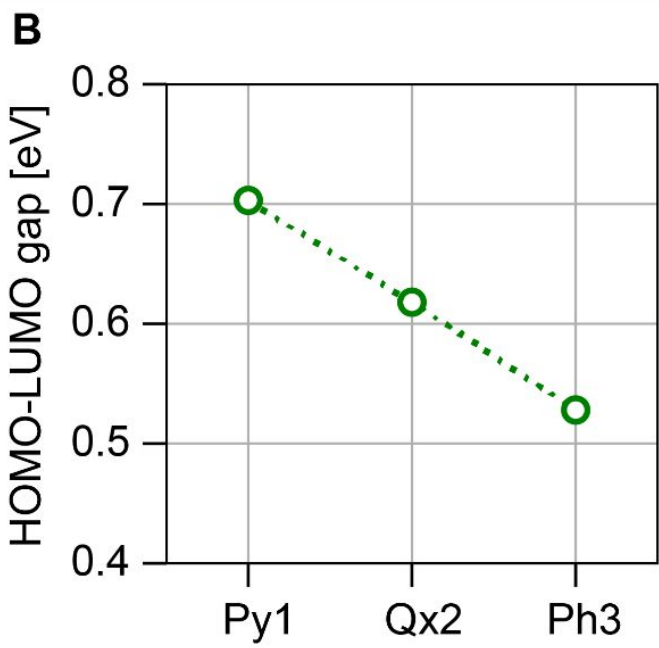

Figure S4. (A) Optimized geometry of Py1, Qx2, and Ph3 with pyramid Au clusters for the L states. (B) HOMO-LUMO gaps of molecular junctions. 
Table S2. HOMO, LUMO energy, and HOMO-LUMO gaps of Py1, Qx2, and Ph3 molecular junctions

\begin{tabular}{ccccc}
\hline Molecular junctions & HOMO $[\mathrm{eV}]$ & LUMO $[\mathrm{eV}]$ & LUMO+1 $[\mathrm{eV}]$ & HOMO-LUMO gap [eV] \\
\hline $\mathrm{Au} / \mathrm{Py} 1 / \mathrm{Au}$ & -4.206 & -3.503 & -2.616 & 0.703 \\
$\mathrm{Au} / \mathrm{Qx} 2 / \mathrm{Au}$ & -4.227 & -3.609 & -2.640 & 0.618 \\
$\mathrm{Au} / \mathrm{Ph} 3 / \mathrm{Au}$ & -4.267 & -3.739 & -2.671 & 0.528 \\
\hline
\end{tabular}




\section{Additional STM-BJ result}

Figure S5 shows the result of another STM-BJ experiment performed using a current amplifier with a gain of $1000 \mathrm{nA} / \mathrm{V}$ and a fixed bias voltage of $100 \mathrm{mV}$. The inset of Figure S5A shows the typical conductance traces of the Py1 molecular junction. We measured and compiled 4150 conductance versus tip displacement curves into 2D maps, as shown in Figure S5A. Here, the relative tip-substrate displacement was set to zero when the conductance was $0.5 G_{0}$. In this histogram, the counts of the highly conductive state begin after the breakage of the Au atomic contact at a conductance of $1.0 G_{0}$. An analysis of the conductance measurements (Figure S5B) shows the double conductance states in the Py1 molecular junction, as outlined in the main text. 

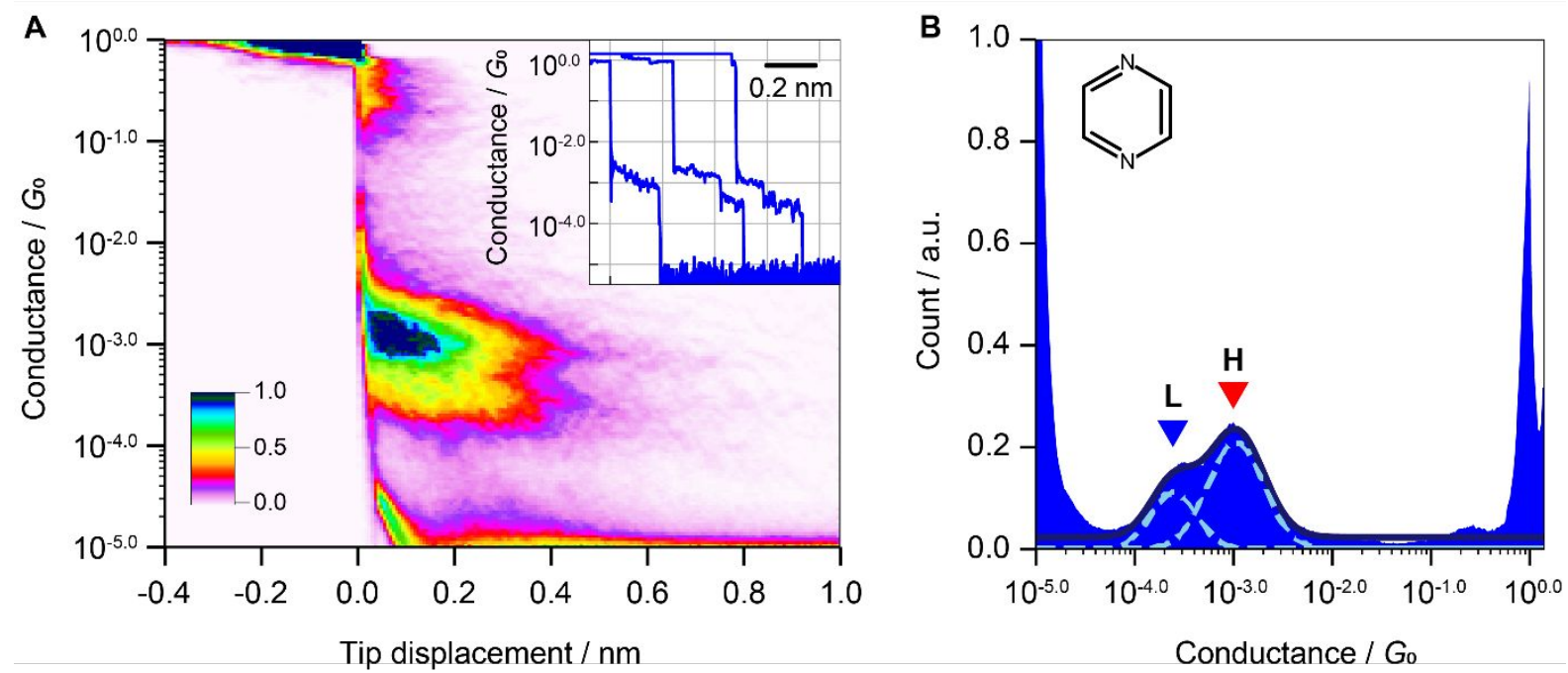

Figure S5. (A) 2D histograms of conductance versus tip displacement traces at the bias voltage of $100 \mathrm{mV}$ for Py1. A linear X-bin size of $6.47 \mathrm{pm}$ was used. The log-scaled Y-bin size of $\Delta \log \left(G / G_{0}\right)$ was 0.0162 . The inset is an example of the conductance traces. (B) Conductance histogram of Py1 created with a log-scaled bin size of $\Delta \log \left(G / G_{0}\right)$ of 0.0167 . 


\section{Analysis of the Junction Length}

Analysis of the tip displacement

The conductance traces were fitted with multiple sigmoid functions, as shown in Equation

(2) of the main text, to evaluate the tip displacement where the conductive states change to other states. The typical conductance traces in the Py1 and Qx2 junctions were well fitted using these functions, as shown in Figures S6A and S6B. The tip displacement at the transition from the $\mathrm{H}$ to $\mathrm{L}$ states and from the $\mathrm{L}$ state to the breakage of the junctions was defined as the displacement $\left(P_{\mathrm{i}}[\mathrm{nm}]\right)$ at the center of the sigmoid functions. Thus, we fabricated 2D histograms of conductance $\left(G\left(P_{i}\right)\left[G_{0}\right]\right)$ versus $P_{\mathrm{i}}$, where $G\left(P_{i}\right)$ is the conductance at $P_{\mathrm{i}}$, as shown in Figures S6C and S6D. The averaged $P_{i}$ of Figure 5 in the main text was defined within the conductance windows (see Table S3), and $10^{-4.8}-10^{-5.5} G_{0}$ for the $\mathrm{L}_{2}$ state of $\mathrm{Ph} 3$.

\section{Analysis of snap-back distance}

We analyzed the snap-back distance of the $\mathrm{Au}$ electrode to determine the electrode separation. Immediately after the breakage of the Au point contact, a nanogap of finite size was formed owing to the elastic snap-back response of the Au electrodes. ${ }^{9-10}$ The snapback distance can be estimated by calculating the difference in the tip displacement in breaking and forming an $\mathrm{Au}$ contact (Figure S7A). ${ }^{9}$ Figure S6 shows an example of conductance versus tip displacement traces in the breaking and forming process of an $\mathrm{Au}$ contact. As shown in Figure S7, the conductance $(G)$ typically showed a sudden change from $G>1 G_{0}$ to $G<0.5 G_{0}$, and from $G<0.5 G_{0}$ to $G>1 G_{0}$. Therefore, based on the threshold value of $0.5 G_{0}$, the snap-back distance is defined in Figure S7A (see the dotted lines). Figure 
6B shows a histogram of the snap-back distance, which is constructed from 4150 conductance versus tip displacement traces for Py1. The peak value in the histogram was determined to be $0.5 \mathrm{~nm}$, which is consistent with the values in previous studies at room temperature. ${ }^{9}$

A

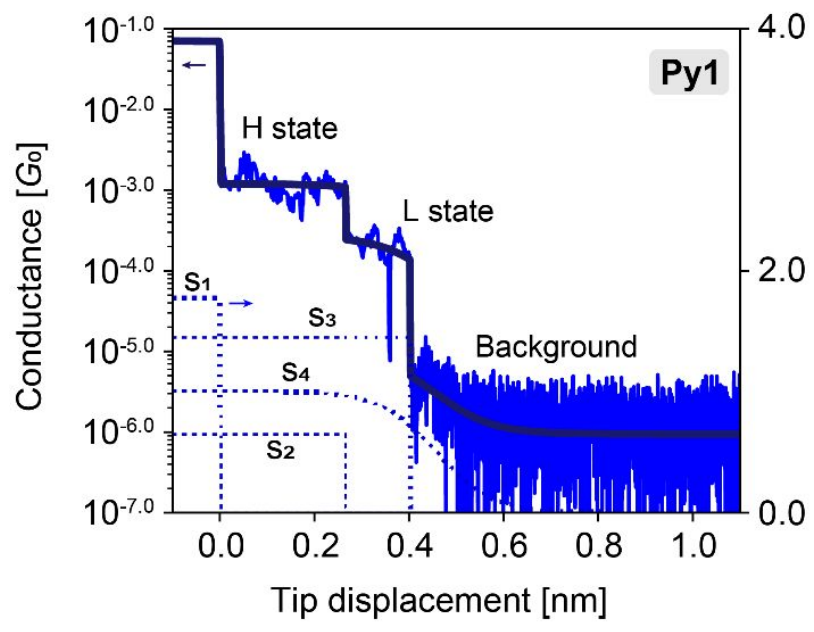

B

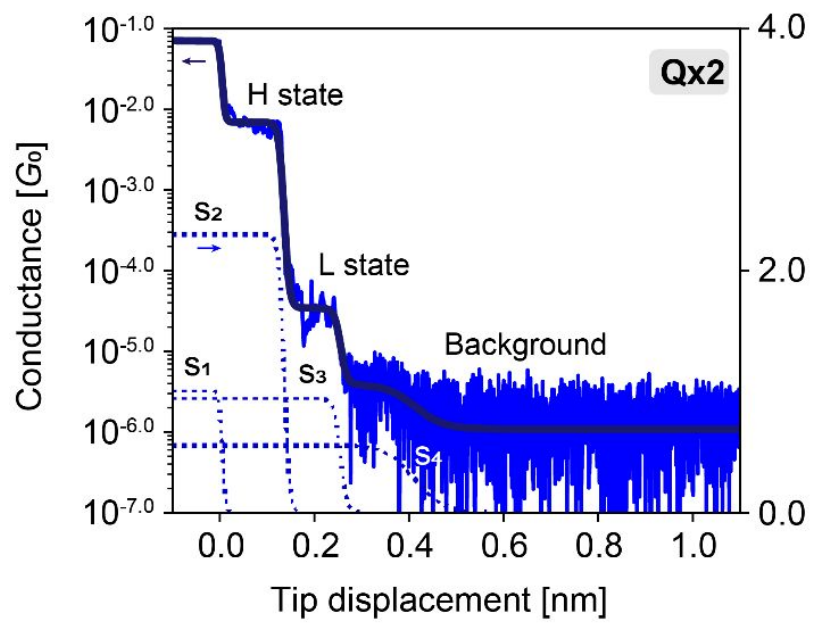

C
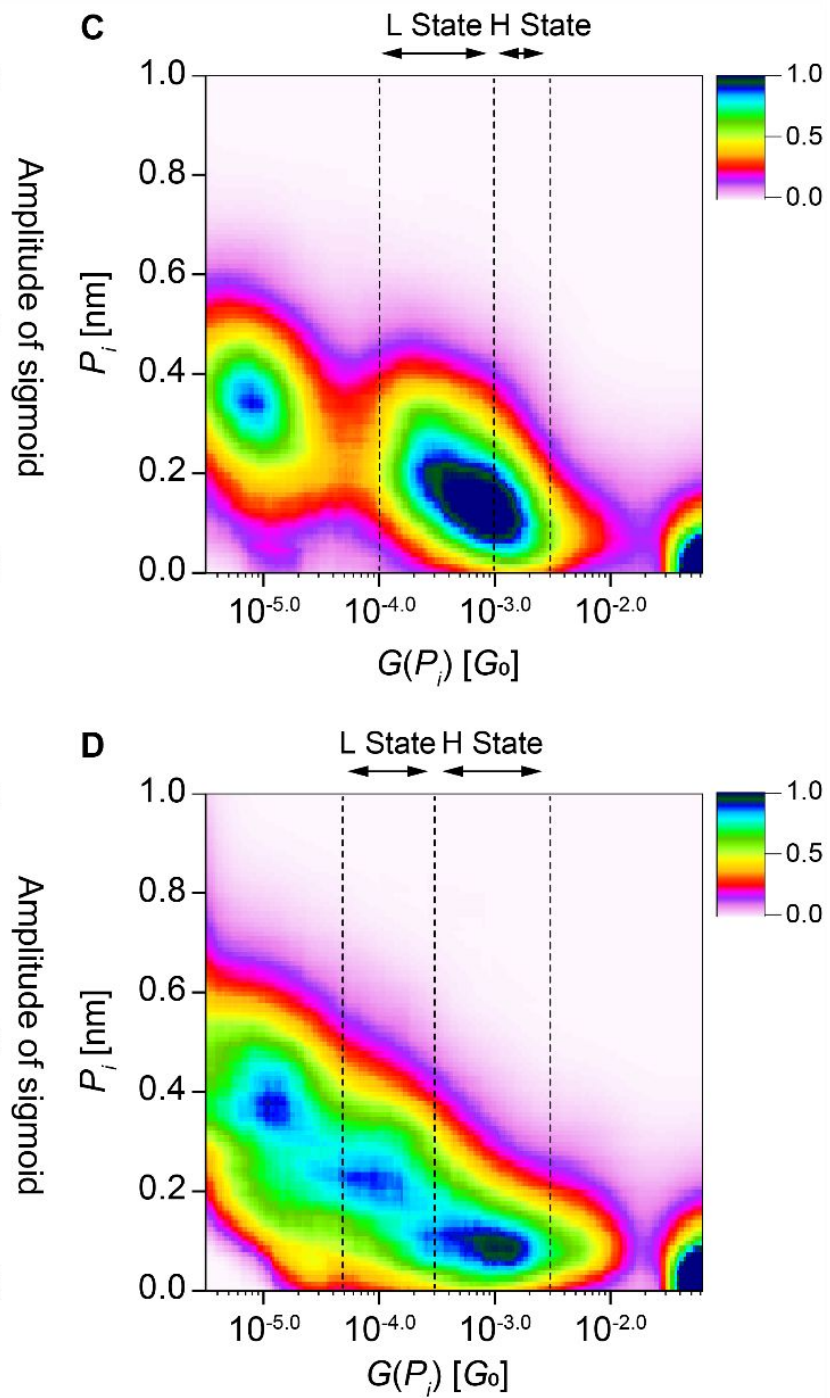

Figure S6. Conductance traces of (A) Py1 and (B) Qx2 fitted with four sigmoid functions. The dotted lines $\left(\mathrm{s}_{1}-\mathrm{s}_{4}\right)$ show the individual fitted lines. (C-D) 2D histograms of conductance versus tip displacement at the center of the sigmoid functions of (C) Py1 and 
(D) Qx2. A logarithmic X-bin size $\Delta \log \left(G / G_{0}\right)$ of 0.0275 and a linear X-bin size of $0.01 \mathrm{~nm}$ were used. 

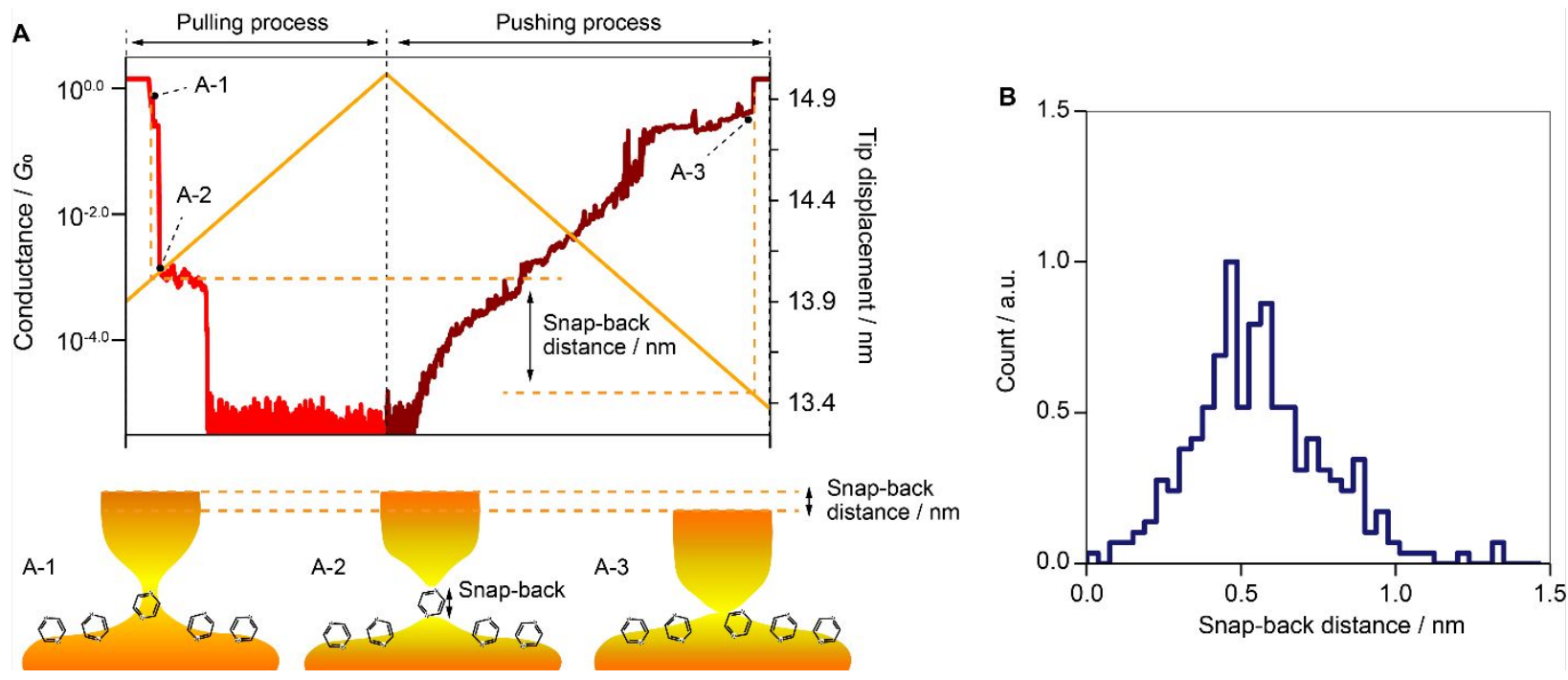

Figure S7. (A) Schematic illustration of the definition of snap-back distance. The red lines represent an example of a conductance versus tip displacement trace during pulling/breaking and pushing/forming of an Au point contact for Py1. (B) Histogram of the snap-back distance for Py1, which was constructed from 4150 conductance traces 
Table S3. Conductance and $P$ Values

\begin{tabular}{cccc}
\hline Molecule & State & $G / \mathrm{m} G_{0}$ & $P / \mathrm{nm}$ \\
\hline Py1 & H & $10^{-3.08 \pm 0.3}$ & $0.16 \pm 0.09$ \\
& L & $10^{-3.70 \pm 0.3}$ & $0.22 \pm 0.12$ \\
Qx2 & H & $10^{-2.15 \pm 0.2}$ & $0.16 \pm 0.03$ \\
& L & $10^{-3.81 \pm 0.9}$ & $0.26 \pm 0.17$ \\
Ph3 & H & $10^{-1.88 \pm 0.5}$ & $0.16 \pm 0.03$ \\
& L & $10^{-3.88 \pm 0.7}$ & $0.27 \pm 0.02$ \\
\hline
\end{tabular}




\section{Statistical $I-V$ Analysis}

For the $I-V$ fitting, the measured $I-V$ curves of the single-molecule junctions were selected based on the preferential conductance windows determined via the statistical analysis of the conductance versus tip displacement traces (Figure 3). Table S3 lists the corresponding conductance windows of the $\mathrm{H}$ and $\mathrm{L}$ states for each molecule. Figure S8 shows examples of the $I-V$ fitting for Py1, Qx2, and Ph3.

Figure S9 shows the histograms of $\Gamma$ for Py1 and Ph3 with and without data selection. For Py1, the $I-V$ curves contained the $\mathrm{H}$ and $\mathrm{L}$ states in the same dataset obtained using the current amplifier with a gain of $1000 \mathrm{nA} / \mathrm{V}$ (see Figure 6A). Therefore, the $I-V$ curves were fitted by two Gaussian distributions without data selection (Figure S9A), whereas the $I-V$ curves within the conductance windows of the $\mathrm{H}$ and L states (Table S3) were fitted by a single Gaussian distribution (Figure S9B). For Qx2 and $\mathrm{Ph} 3$, the $\mathrm{H}$ and L states were separately measured using current amplifiers with gains of 1000 and $10 \mathrm{nA} / \mathrm{V}$, respectively. Figure S9C shows an example of the $I-V$ fitting of the $\mathrm{H}$ state for $\mathrm{Ph} 3$ with and without data selection (Figure 6C). For the data selection of the $\mathrm{H}$ state, the conductance window listed in Table S3 was used.

We confirmed that, for $\mathrm{Py} 1$ and $\mathrm{Ph} 3$ as well as Qx2, the peak positions of $\Gamma$ and $\varepsilon$ obtained via Gaussian fitting were the same with and without data selection (Figures S9D, S9E, and S9F). 

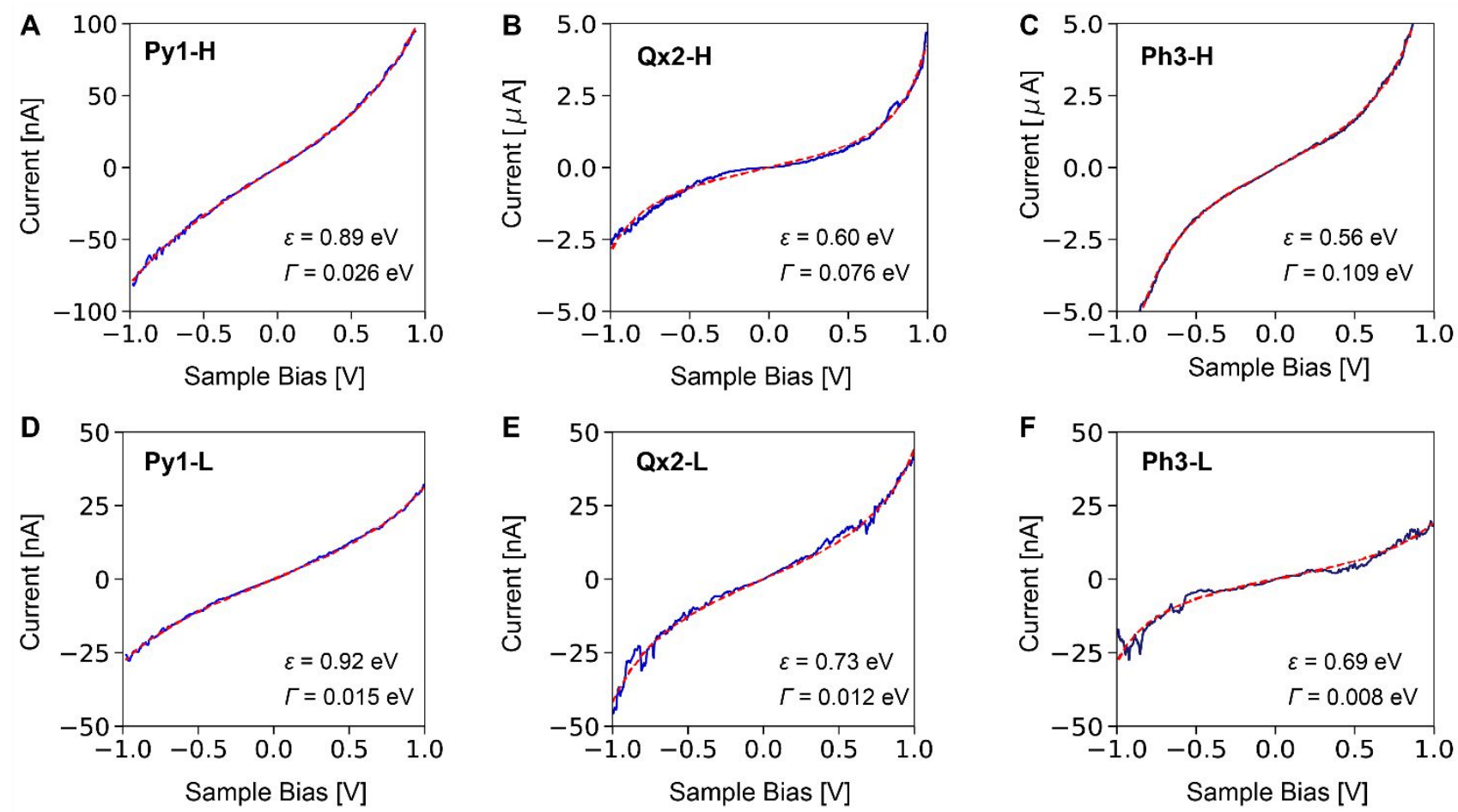

Figure S8. Examples of fitting of $I-V$ curves to equation (3) for the $\mathrm{H}$ and $\mathrm{L}$ state of Py1, Qx2, and $\mathrm{Ph} 3$. Blue and red lines correspond to experimental data and fitting result, respectively. Each $\varepsilon$ and $\Gamma$ values obtained by the fitting are shown in the lower right corner of the graphs. 

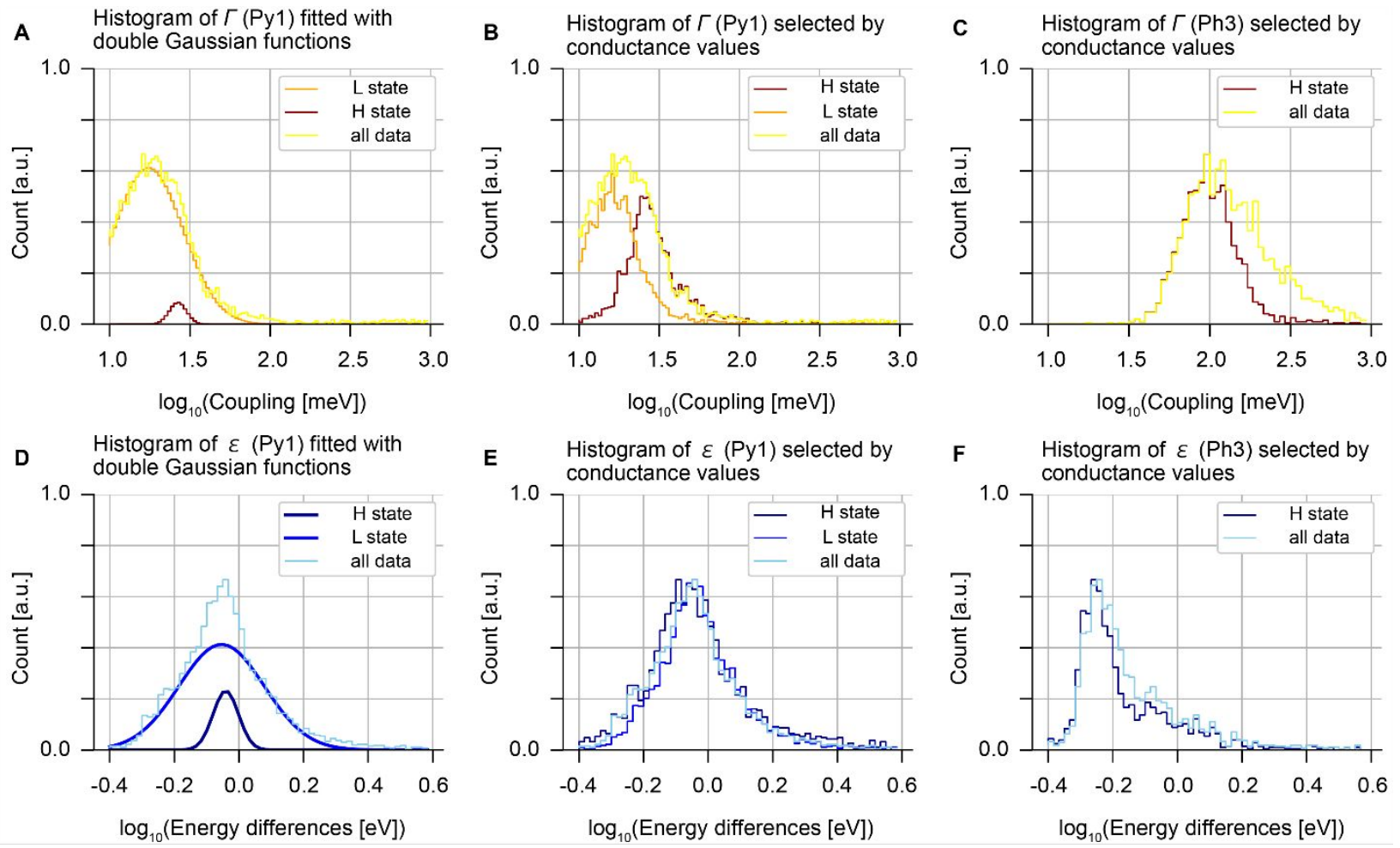

Figure S9. Histograms of $\Gamma$ obtained by the fitting of the $I-V$ curves with and without data selection for $(\mathrm{A}, \mathrm{B})$ the $\mathrm{H}$ and $\mathrm{L}$ states of Py1 and $(\mathrm{C})$ the $\mathrm{H}$ state of Ph3. Histograms of $\varepsilon$ obtained with and without data selection for (D,E) the $\mathrm{H}$ and L states of Py1 and (F) the $\mathrm{H}$ state of Ph3. The bin sizes of $\Delta \log (\Gamma)$ and $\Delta \log (\varepsilon)$ are 0.033 and 0.017 , respectively. 
Table S4. Conductance Windows for the Selection of the I-V Curves

\begin{tabular}{ccc}
\hline Molecule & State & Window \\
\hline Py1 & H & $10^{-3.0}-10^{-2.8} G_{0}$ \\
& L & $10^{-4.0}-10^{-3.0} G_{0}$ \\
Qx2 & H & $10^{-3.8}-10^{-2.8} G_{0}$ \\
& L & $10^{-4.5}-10^{-3.9} G_{0}$ \\
Ph3 & H & $10^{-2.9}-10^{-1.9} G_{0}$ \\
& L & $10^{-4.8}-10^{-3.0} G_{0}$ \\
\hline
\end{tabular}



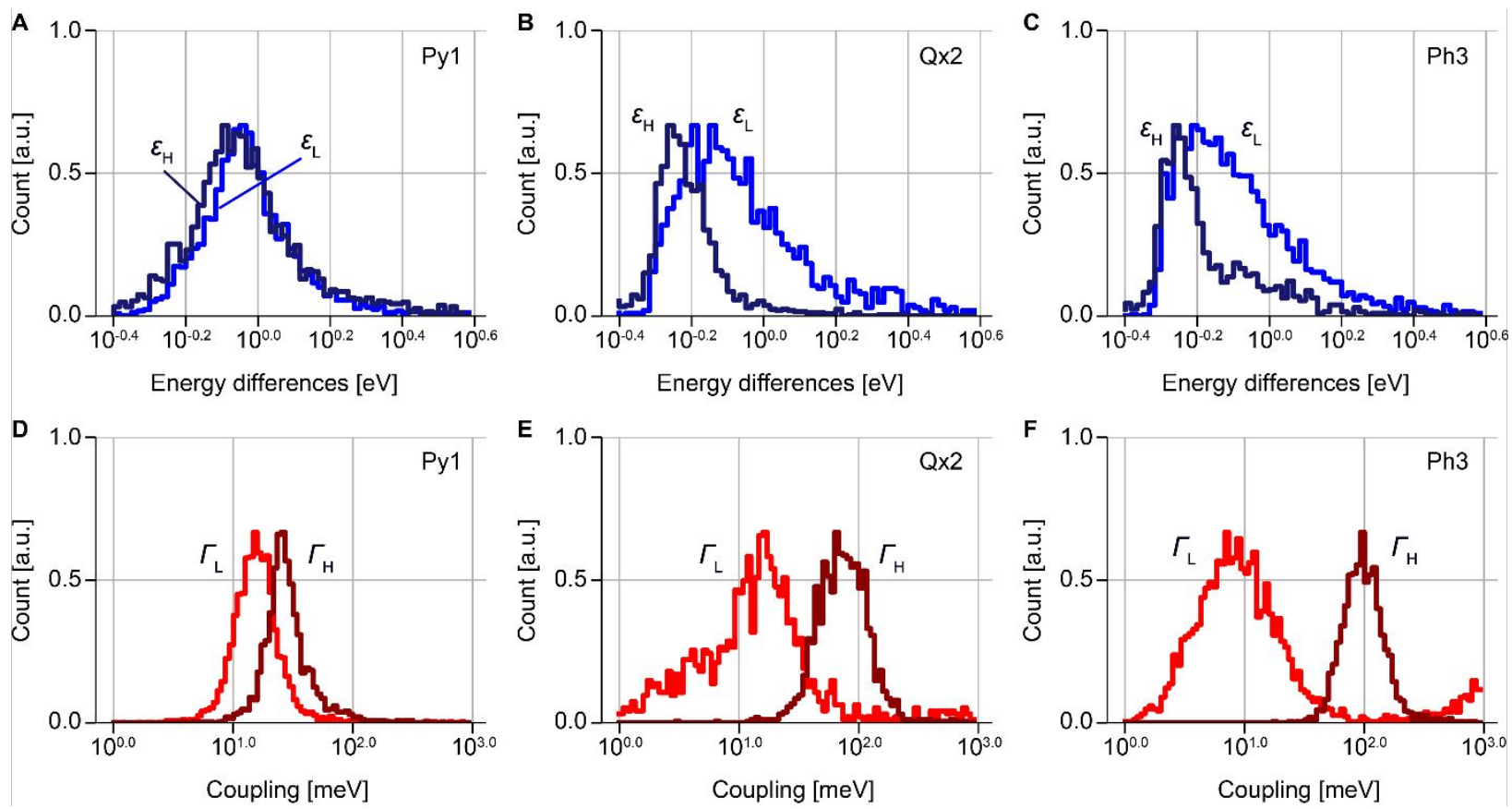

Figure S10 Log-binned histograms of (A-C) $\varepsilon$, and (D-F) $\Gamma$ for Py1, Qx2, and Ph3 where the $\log$-scaled bin sizes of $\Delta \log (\varepsilon)$ and $\Delta \log (\Gamma)$ were 0.0166 and 0.0333 , respectively. 


\section{REFERENCES}

(1) Frisch, M. J.; Trucks, G. W.; Schlegel, H. B.; Scuseria, G. E.; Robb, M. A.; Cheeseman, J. R.; Scalmani, G.; Barone, V.; Petersson, G. A.; Nakatsuji, H.; Li, X.; Caricato, M.; Marenich, A. V.; Bloino, J.; Janesko, B. G.; Gomperts, R.; Mennucci, B.; Hratchian, H. P.; Ortiz, J. V.; Izmaylov, A. F.; Sonnenberg, J. L.; Williams-Young, D.; Ding, F.; Lipparini, F.; Egidi, F.; Goings, J.; Peng, B.; Petrone, A.; Henderson, T.; Ranasinghe, D.; Zakrzewski, V. G.; Gao, J.; Rega, N.; Zheng, G.; Liang, W.; Hada, M.; Ehara, M.; Toyota, K.; Fukuda, R.; Hasegawa, J.; Ishida, M.; Nakajima, T.; Honda, Y.; Kitao, O.; Nakai, H.; Vreven, T.; Throssell, K.; Montgomery, J. A., Jr.; Peralta, J. E.; Ogliaro, F.; Bearpark, M. J.; Heyd, J. J.; Brothers, E. N.; Kudin, K. N.; Staroverov, V. N.; Keith, T. A.; Kobayashi, R.; Normand, J.; Raghavachari, K.; Rendell, A. P.; Burant, J. C.; Iyengar, S. S.; Tomasi, J.; Cossi, M.; Millam, J. M.; Klene, M.; Adamo, C.; Cammi, R.; Ochterski, J. W.; Martin, R. L.; Morokuma, K.; Farkas, O.; Foresman, J. B.; Fox, D. J. Gaussian 16, Revision C.01; Gaussian, Inc.: Wallingford CT, 2016.

(2) Bauernschmitt R.; Ahlrichs, R. Treatment of electronic excitations within the adiabatic approximation of time-dependent density functional theory. Chem. Phys. Lett. 1996, $256,454-464$.

(3) Isshiki, Y.; Fujii, S.; Nishino, T.; Kiguchi, M. Fluctuation in interface and electronic structure of single-molecule junctions investigated by current versus bias voltage characteristics. J. Am. Chem. Soc. 2018, 140, 3760-3767.

(4) Perdew, J. P.; Ernzerhof, M.; Burke, K. Rationale for mixing exact exchange with density functional approximations. J. Chem. Phys. 1996, 105, 9982-9985.

(5) Perdew, J. P.; Burke, K.; Ernzerhof, M. Generalized gradient approximation made simple. Phys. Rev. Lett. 1996, 77, 3865-3868.

(6) Delley, B. From molecules to solids using the DMol3 approach. J. Chem. Phys. 2000, $113,7756-7764$. 
(7) Delley, B. An all-electron numerical method for solving the local density functional for polyatomic molecules. J. Chem. Phys. 1990, 92, 508-517.

(8) Grimme S. Density functional theory with London dispersion corrections. Wiley Interdisciplinary Reviews: Comput. Mol. Sci. 2011, 1, 211-228.

(9) Quek, S. Y.; Kamenetska, M.; Steigerwald, M. L.; Choi, H. J.; Louie, S. G.; Hybertsen, M. S.; Neaton, J. B.; Venkataraman, L. Mechanically controlled binary conductance switching of a single-molecule junction. Nat. Nanotechnol. 2009, 4, 230-234.

(10) Van Ruitenbeek, J. M.; Alvarez, A.; Pineyro, I.; Grahmann, C.; Joyez, P.; Devoret, M. H.; Esteve, D.; Urbina, C., Adjustable nanofabricated atomic-size contacts. Rev. Sci. Instrum. 1996, 67, 108-111. 